\title{
ANÁLISIS ESTRATIGRÁFICO DE LA FORMACIÓN SAN GREGORIO (PÉRMICO INFERIOR), EN LOS TESTIGOS DEL POZO CERRO LARGO SUR N4 (DI.NA.MI.GE.), URUGUAY
}

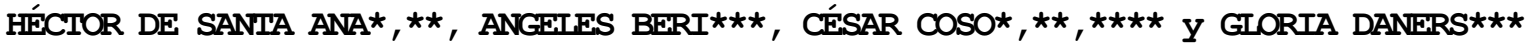

\begin{abstract}
STRATIGRAPHIC ANALYSIS OF THE SAN GREGORIO FORMATION (LOWER PERMIAN), BASED IN CORES FROM THE CERRO LARGO SUR No4 WELL (DI.NA.MI.GE.), 4 well corresponding to the claystones, with levels of diamictites; unit II. composed of diamictites, sandstones and rithmites; and unit III, composed of gray shale and siltstones. The unit II, where the gravitational flows are predominant, was characterized as resedimentation facies. Moreover, three palynological associations were defined, correlated with the proposed Zones of the Chaco-Paraná Basin (Argentina) and Paraná Basin (Brazil). A Lower Permian age is stated.
\end{abstract}

Keywords: Stratigraphy, Norte Basin, Permian, San Gregorio Formation, Palynology, Uruguay.

\begin{abstract}
RESUMEN En depósitos correspondientes a la Formación San Gregorio, fueron reconocidas tres unidades faciológicas de naturaleza subacuatica en los testigos de la perforación DCLS $\mathrm{N}^{\circ}$ 4: unidad I de pelitas grises y arcillitas negras, con niveles de diamictitos; unidad II de diamictitos, areniscas y ritmitos; y unidad III de pelitas y limohtas grises. La unidad II, en donde los flujos gravitacionales son predominantes, fue caracterizada como facies de resedimentación. Âl mismo tiempo, fueron definidas tres asociaciones palinológicas, estableciéndose correlaciones con las zonaciones propuestas para la región. Cuenca Chaco-Paraná (Argentina) y Cuenca del Paraná (Brasil), proponiéndose una edad Pérmico Inferior.
\end{abstract}

Palabras clave: Estratigrafía, Cuenca Norte, Pérmico, Formación San Gregorio, Palinología, Uruguay.

INTRODUCCIÓN Se realiza un estudio faciológico de detalle (lito-bio-cronoestratigráfico) tendiente a caracterizar los procesos sedimentarios actuantes en el sector este de la Cuenca Norte Uruguaya. El mismo corresponde íntegramente a los sedimentos atribuidos a la Formación San Gregorio, en base a la información disponible en los testigos de la perforación DCLS No 4 (DI.NA.MI.GE) (Fig. 1).

Los resultados obtenidos son coherentes con las diversas reconstrucciones paleogeográficas postuladas por diferentes autores a nivel local y regional. La predominancia de sistemas depositacionales marinos de relativa profundidad, la presencia de depósitos de resedimentación, el control glacioeustático de la sedimentación, la comparación y correlación regional de las asociaciones de palinomorfos, como también algunas consideraciones sobre la edad de la Formación San Gregorio, se explicitarán en este trabajo.

ANTECEDENTES El origen glacial de los sedimentos de la Formación San Gregorio ha sido establecido desde principios de siglo por varios autores (Walther 1924, Terra Arocena 1926, Falconer 1937), quienes correlacionaban y señalaban ese origen basados en los estudios que había iniciado White (1908) en Brasil. Desde ese momento, los estudios de la Formación San Gregorio fueron abordados bajo la óptica de depósitos correspondientes a ambientes glaciales, periglaciales (lacustres) y fluvio-glaciales, hasta que, recientemente, se establecen por algunos autores, para esta unidad, litologías vinculadas a procesos de remoción en masa y corrientes de turbidez (Ferrando \& Andreis 1986, Ferrando \& Montaña 1987, de Santa Ana 1989).
Los estudios recientes de sedimentación glacia, ya sea en depósitos holocénicos como en registros más antiguos, demuestran la abundancia de facies de resedimentación, asociadas e intercaladas a facies marinas y transicionales (Eyles et al 1983, Miall 1983).

Los depósitos directamente vinculados a la sedimentación glacial tienen un muy bajo potencial de preservación (Miall 1985), producto fundamentalmente de la acción del deshielo. Los flujos gravitacionales serían los verdaderos y efectivos agentes depositacionales, mientras que los glaciales desempeñarían la función de agentes de transporte y aporte de terrígenos (Gama Jr. et al 1989).

MATERIALES Y MÉTODOS El método empleado en el análisis del perfil de pozo se apoya en la aplicación de las técnicas de análisis de facies y modelos de facies propuestos por Walker (1984). El análisis sedimentológico de algunas unidades definidas en los testigos del pozo fueron identificadas siguiendo el esquema clasificatorio propuesto por Eyles et al (1983), la que puede resumirse en el siguiente cuadro: $\mathrm{D}=$ Diamictito; $\mathrm{D}_{\mathrm{m}}=$ matriz-soportado; $\mathrm{D}_{\mathrm{c}}=$ clastosoportado; $\mathrm{D}_{\mathrm{rm}}=$ masivo; $\mathrm{D}_{\mathrm{s}}=$ estratificado; $\mathrm{S}=$ Arenisca; $\mathrm{S}_{\mathrm{r}}$ $=$ con ripples; $\mathrm{S}_{\mathrm{h}}=$ con laminación horizontal; $\mathrm{S}_{\mathrm{m}}=$ masiva; $\mathrm{S}_{\mathrm{g}}=$ con gradación; $\mathrm{F}=$ Finos; $\mathrm{F}_{\mathrm{m}}=$ masivos; $\mathrm{F}_{1}=$ laminados; $\mathrm{F}_{-\mathrm{d}}=$ con dropstones.

El estudio palinológico se realizó a partir de un muestreo sistemático en las unidades pelíticas oscuras debido a la presencia de materia orgánica. Se tomaron un total de 14 muestras desde los $-72 \mathrm{~m}$ a los $-7 \mathrm{~m}$ y fueron procesadas con los métodos comunes para palinología, resultando todas ellas fértiles.

* $\quad$ A.N.C.A.P., División de Investigación y Desarrollo, Avenida Brigadero Gral. Juan A. Lavalleja s/n y Paysandú, Casilla Postal 11000,1090

Montevideo, Uruguay

** Departamento de Geología, Facultad de Ciencias, Tristán Narvaja 1674,11200, Montevideo, Uruguay

*** Departamento de Paleontología, Facultad de Ciencias, Tristán Narvaja 1674,11200 Montevideo, Uruguay

**** Postgraduando de Instituto de Geociências e Ciências Exatas, Universidade Estadual Paulista, Caixa Postal 178, CEP 13500-970, Rio Claro, SP, Brasil 


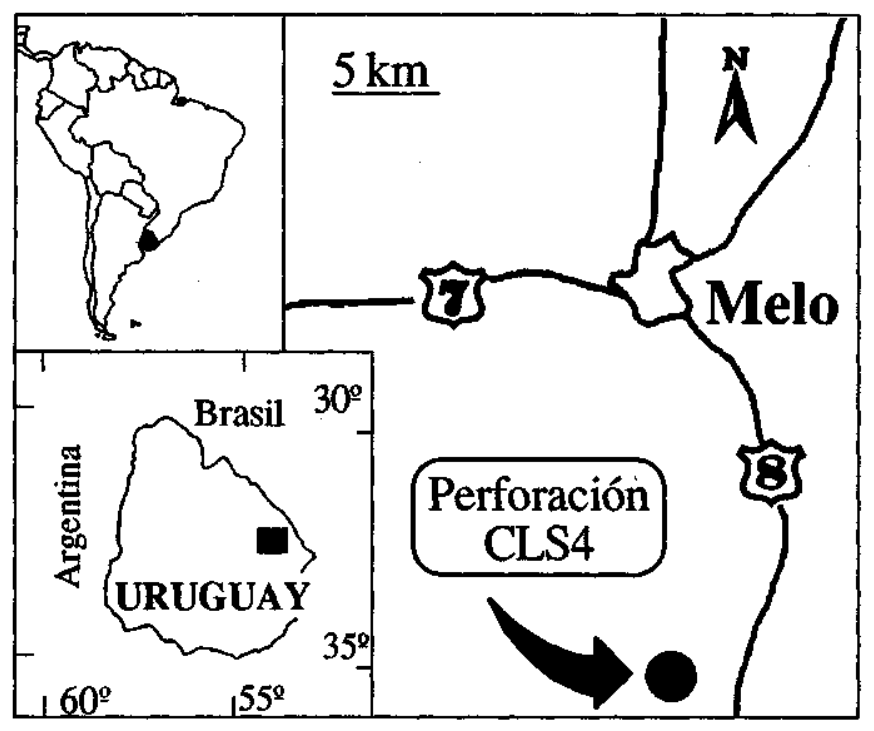

Figura 1 - Ubicación de la perforación DCLS N- 4 Figure 1 - Location of the drill hole DCLS N 4

El número mínimo de palinomorfos que debe contener una muestra paleozoica, para que resulte representativa, es de 200 ejemplares. En todos los niveles se logró llegar a este número, o muy cerca de él, salvo en los sectores correspondientes a los $-36,-38$ y $-55 \mathrm{~m}$ en los que resultó una palino- flora muy pobre, por lo que no serán aquí considerados.

Los diferentes taxones fueron separados en seis grandes grupos de acuerdo a su morfología, para analizar su comportamiento a lo largo del perfil.

\section{RESULTADOS Análisis Sedimentológico En}

el perfil de la perforación analizada (Fig. 2) fueron caracterizadas tres unidades faciológicas que se detallan a continuación, utilizándose la propuesta de nomenclatura de litofacies de Eyles et al. (1983).

Unidad I Descripción Esta unidad predomina hacia el tercio inferior del perfil y está caracterizada por lutitas grises oscuras con estratificación media a fina, plano-paralela, y arcillitas negras finamente laminadas, piritosas y con abundante materia orgánica.

Hacia la base se apoya discordantemente sobre el basamento y se intercalan términos métricos a decimétricos (de 78 a $-73 \mathrm{~m}$ ) de diamictitos masivos con matriz psamopelítica con clastos polimícticos de hasta $2 \mathrm{~cm}$, y limoarcillitas grises con granulos y areniscas finas gradadas.

Interpretación Estos depósitos indican un predominio de procesos de decantación, con baja tasa de sedimentación bajo condiciones subacuáticas muy calmas, mal oxigenadas y mal drenadas.

El perfil presenta enriquecimiento de materia orgánica hacia el tope de esta unidad (-62 m), marcando condiciones de mínima energía y máxima restricción para este sector de la cuenca.

Unidad II Descripción Esta unidad es dominante en la sección del pozo desarrollándose en el tercio medio y parte del tercio superior. Ha sido caracterizada íntegramente como facies de resedimentación y desde el punto de vista litológico han sido reconocidos los siguientes términos: a. Diamictitos Los diamictitos no superan espesores del orden métrico, presentan generalmente clastos no mayores de 3-4 cm, de naturaleza polimíctica e inmersos en matriz arenosa o pelítica. Generalmente, los clastos son redondeados; predominan las facies $D_{m-m}$, aunque ocurre algún tipo de organización incipiente de tipo $D_{\mathrm{m}-\mathrm{s}}$ y $\mathrm{D}_{\mathrm{c}-\mathrm{s}}$.

Se desarrollan gradaciones normales y estratificaciones pobremente definidas. Es característica la presencia de estructuras deformacionales, con el desarrollo de figuras contorsionadas.

b. Areniscas Las areniscas varían desde muy gruesas a muy finas en bancos que no superan los $3 \mathrm{~m}$. Presentan estratificación bien definida, y hacia los $-20 \mathrm{~m}$ aparecen estratificaciones cruzadas.

La ocurrencia de estratificación gradacional normal es muy frecuente (de tipo $\mathrm{S}_{\mathrm{g}}$ ). En areniscas de granulometría media fueron observadas laminaciones cabalgantes (climbing ripples).

c. Ritmitos Conjuntamente con los diamictitos, los ritmitos son los sedimentos más conspicuos del perfil, alcanzando espesores de hasta $15 \mathrm{~m}$.

Fueron reconocidos dos tipos de ritmitos predominantes en esta perforación. El primer tipo, donde el carácter rítmico está dado por la facie $\mathrm{F}_{1}$, cuya laminación horizontal separa estratos milimétricos a centimétricos de limos y arcillas, con contacto neto, siendo esa separación a veces con facies arenosas de tipo $\mathrm{S}_{\mathrm{h}}$. Un segundo tipo de ritmitos caracterizados por la intercalación de facies $\mathrm{S}_{\mathrm{g}} / \mathrm{S}_{\mathrm{r}}($ ?), y la propia $\mathrm{F}$, cuya distinción es nítida por la presencia de gradación. $\mathrm{La}$ ocurrencia de facies arenosas con ripples $\left(\mathrm{S}_{\mathrm{r}}\right)$ es dudosa. Son muy comunes las deformaciones plásticas sindepositacionales y la aparición de dropstones. Varios de los testigos analizados presentan fallas sindepositacionales asociadas a los ritmitos.

Interpretación Integran esta unidad, depósitos generados por flujos gravitacionales, englobando bajo este concepto desde los deslizamientos de material cohesivo hasta los flujos turbulentos de baja concentración, incluyendo los flujos de masa de viscosidad variable (Middleton \& Hampton 1973). Son predominantes, desde los -60 hasta los $-12 \mathrm{~m}$, una serie de mecanismos destructivos representados a través de facies de resedimentación.

Relacionados con el predominio de estos procesos, es oportuno realizar las siguientes consideraciones: a. En la generación de los depósitos diamictíticos operan flujos de alta densidad y viscosidad relativa, los que sujetos a condiciones confinadas o no, pueden producir facies $\mathrm{D}_{\mathrm{c}-\mathrm{s}}$, $D_{m-s}$ en el primer caso, o facies $D_{m}-m$ las que se asocian a desbordamientos en situaciones no confinadas, indicando que estas facies son originadas por flujos de barro (mud flows) o flujos de detritos (debris flows). Similares procesos ocurrieron en la generación de los diamictitos presentes en la unidad I.

b. Las facies arenosas $S_{g}$ y $S_{r}$ involucran disminución de la viscosidad, por lo que se admite disminución de la velocidad debido a la pérdida de concentración, tal vez a causa de una menor pendiente de la superficie depositacional. De esa forma se materializan algunos términos Tabc y Tab de la secuencia de Bouma (1962).

c. Las facies de areniscas masivas $\mathrm{S}_{\mathrm{m}}$ pueden estar vinculadas a flujos licuefactos (liquefied flows), es decir, flujos de carácter laminar y de alta densidad (Lowe 1979). La asociación con turbiditas arenosas y con facies pelíticas evidencia que la rápida desaceleración del flujo pudo haber ocurrido en el pasaje de condiciones confinadas a no confinadas, en la porción terminal de los canales subacu- 


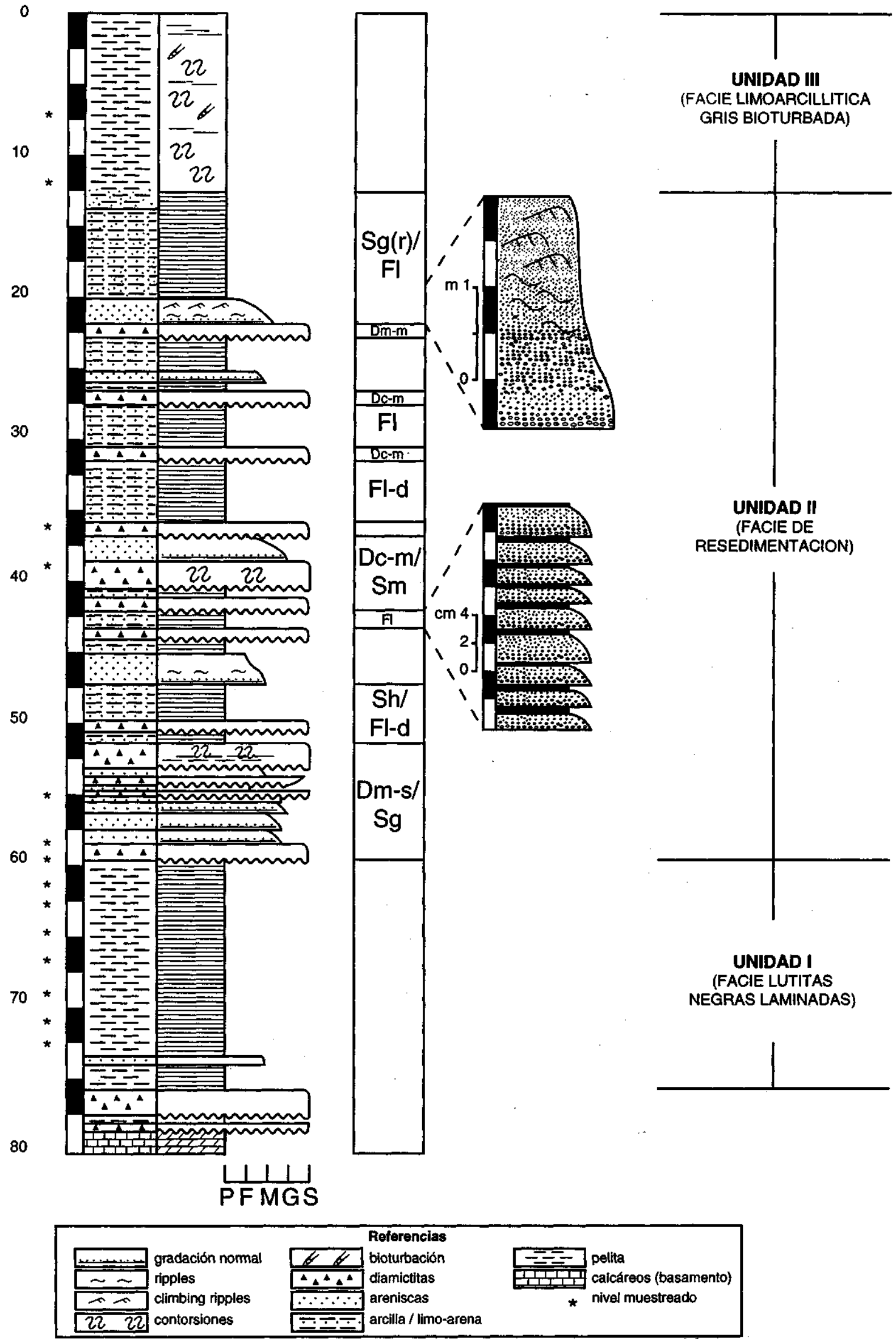

Figura 2 - Perfil estratigráfico de la perforación estudiada Figure 2 - Stratigraphic section of the studied drill hole 
osos. Esto implica desaceleración abrupta, depositándose muy rápidamente la carga sedimentaria (Gama Jr. et al 1989).

d. Las facies de ritmitos se vinculan a las comentes de turbidez de baja densidad. Estos son flujos gravitacionales turbulentos de baja velocidad y, por lo tanto, caracterizados por el transporte de las fracciones arenosas más finas y predominantemente limos y arcillas. Las turbiditas se presentan como ritmitos con variaciones en la secuencia vertical de estructuras entre las cuales las más abundantes son $\mathrm{Ta} /(\mathrm{e}), \mathrm{Tbcd} /(\mathrm{e})$.

De alguna manera y asociado tanto a los diamictitos como a los ritmitos, uno de los aspectos marcantes es la abundancia de convoluciones y deformaciones. Inclusive han podido ser observados en los depósitos con deslizamientos las formas sindepositacionales.

Unidad III Descripción Se desarrolla hacia el tercio superior de la sección estudiada. Presenta una potencia de 12 $\mathrm{m}(-12$ a $0 \mathrm{~m})$ y está caracterizada por limolitas y pelitas de color gris claro, micáceas y piritosas, con estratificación mal definida, fuertemente bioturbadas.

Interpretación $\mathrm{Se}$ registra un momento de nulo aporte de material por tracción, predominando los depósitos de decantación, desarrollados en ambiente semi-restricto y con importante actividad biológica. Estas condiciones evidenciarían aparente calma tectónica, climática y eustática para este sector de la cuenca.

Análisis Palinológico Fueron halladas un total de 64 especies de esporomorfos (Beri \& Daners ms). El análisis de la distribución de los diferentes grupos en el perfil (Fig. 3) permite realizar las siguientes consideraciones:

- tanto las esporas triletes apiculadas, como los granos de polen bisacados y estriados, son escasos, siendo que estos dos últimos se distribuyen homogéneamente en todos los niveles estudiados.

- os palinomorfos que tienen representación relativamente abundante, son las esporas triletes lisas, las cingulizonadas y los granos de polen monosacados.

- algas del tipo Botryococcus asignadas a ambientes de agua dulce o salobre (Stewart 1983) tienen distribución bastante homogénea en las muestras estudiadas.

- de acuerdo con la proporción de los diferentes grupos, existen tres asociaciones palinológicas que son: 1. de -72 hasta $-66 \mathrm{~m}$, en donde las esporas triletes lisas presentan el mayor porcentaje, siguiéndolas en abundancia las esporas cingulizonadas y los monosacados que se presentan en cantidades similares, las especies más importantes son Punctatisporites gretensis, Caheniasaccites ovatus, Potonieisporites brasiliensis, Vallatisporites arcuatus y Cristatisporites morungavensis; 2. hacia los $-64 \mathrm{~m}$, los monosacados pasan a ser los más abundantes $(60 \%)$, le siguen las triletes lisas y las cingulizonadas, que tienen escasa representación $(5 \%)$, siendo las especies más abundantes Plicatipollenites malabarensis, Potonieisporites brasiliensis y Potonieisporites methoris: 3. a partir de los 62 hasta los $-7 \mathrm{~m}$, las cingulizonadas son las más abundantes, las triletes lisas en menor medida y los granos monosacados son muy escasos. Las esporas triletes apiculadas están presentes en estos niveles pero en porcentajes bajos. Las especies más representativas son Lundbladispora braziliensis, L. riobonitensis, Cristatisporites morungavensis y Vallatisporites arcuatus.

El contenido palinológico asociado a facies de resedimentación, tiene escaso valor como indicador paleoambiental, dado que el material sedimentario es alóctono a la cuenca; sin embargo, es en este caso considerado como buen indicador cronoestratigráfico y climático.

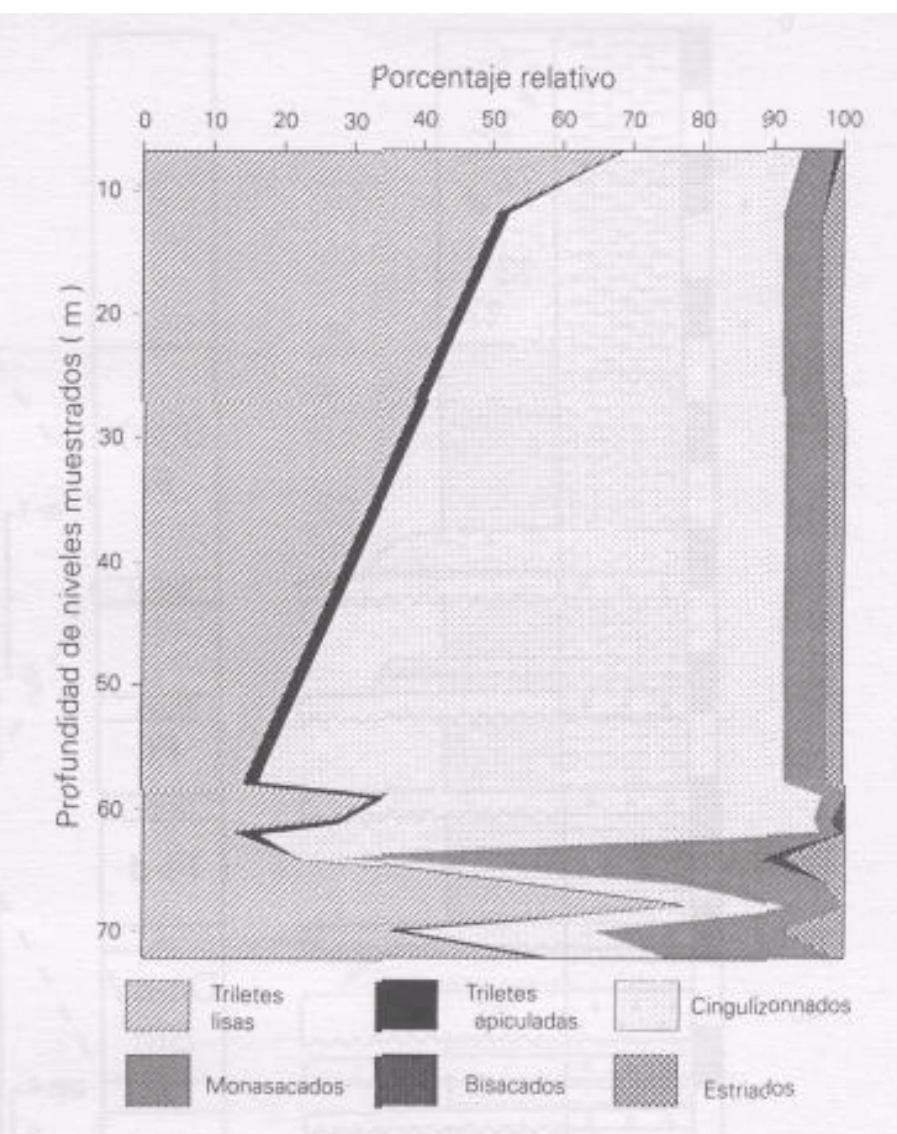

Figura 3- Abundância relativa de los grupos de esporomorfos presentes en los niveles muestrados

Figure 3 - Relative abundance of the sporomorphs presen in the sampled levels

DISCUSIÓN Y CONCLUSIONES Las facies de resedimentación generadas por flujos gravitacionales caracterizan parte de los procesos sedimentarios vinculados a la depositación de la Formación San Gregorio. Los mismos están directamente vinculados a variaciones del nivel del mar (agua de deshielo) y a una mayor tasa de sedimentación. Esto último se correlaciona con el relativo decrecimiento de material polínico observado. Se constata, entonces, el ingreso a la cuenca de abundante material glacial continental removilizado del área depositacional original por la acción de deshielo.

Los procesos de resedimentación se relacionarían con una fusión parcial o total del hielo en condiciones climáticas más benignas y con un mayor gradiente fisiográfico del área de aporte compensado por el ascenso del nivel del mar. Por otro lado, surge del análisis palinológico el predominio y la abundancia relativa de esporas cingulizonadas, la disminución de los granos de polen monosacados y la aparición de esporas triletes apiculadas, lo que demostraría el pasaje de condiciones muy frías y secas (de -72 a $-63 \mathrm{~m}$ ) a condiciones frías menos rigurosas y húmedas (de -62 a $-7 \mathrm{~m}$ ), con desarrollo de vegetación de tierras bajas parcial o totalmente inundadas por largos periodos (Remy 1975).

De la comparación de la asociación de especies de palinomorfos encontrada en esta unidad, con las zonaciones propuestas para la Cuenca Chacoparanense en Argentina por Russo et al. (1980) y Archangelsky \& Vergel (1991) y para la Cuenca del Paraná en Brasil por Marques-Toigo (1988), se desprende que: a. la palinoflora presente en esta perforación se correlaciona con la parte inferior de la Zona Cristatisporites, de edad pérmico inferior o la parte alta de la Zona Potonieisporites - Lundbladispora (límite Carbonífero 
Superior - Pérmico Inferior) de la propuesta argentina; b. en lo referente a la zonación brasileña, se correlaciona con la Zona Cannanoropollis korbaensis, (Sakmariano Superior - Kunguriano Inferior)

Cabe señalar que la gran extensión y continuidad de estos depósitos subacuáticos en toda la Cuenca Norte, de Paraná y del Chaco (Barros Franca \& Potter 1991, Russo et al. 1980, de Santa Ana 1989), demuestra la presencia de una cuenca de importante magnitud regional, dificilmente concebible en ámbitos no marinos. En este sentido, la presencia de algas de agua dulce o salobre del tipo Botryococcus supone una muy baja salinidad del cuerpo de agua.
Por otra parte, demuestrase la correspondencia palinológica, sedimentológica y estratigráfica en relación con la evolución climática y los procesos de transporte y depositación de la cuenca, así como los factores y condicionantes generadores de las diferentes facies sedimentarias reconocidas.

Agradecimientos Deseamos agradecer a los Profs. Jorge Spoturno y Jorge Da Silva de la DI.NA.MI.GE., por el acceso a los materiales de la perforación estudiada; y al Prof. Dr. S. Archangelsky, por la lectura y sugerencias realizadas sobre el manuscrito.

\section{REFERENCIAS BIBLIOGRÁFICAS}

ARCHANGELSKY, S. \& VERGEL, M. 1991. El Sistema Pérmico en la Rep. Argentina y en la República Oriental del Uruguay. In: CONGR. INTERN. ESTRATIGR. Y GEOL. DEL CARBONIR-PERMICO, 12 y ACAD. NAC. DE CIEN. DE CÓRDOBA. Buenos Aires, 1991. p. 40-44. (Pre-print).

BARROS FRANCA, A. \& POTTER, P.E. 1991. Stratigraphy and reservoir potencial of glacial deposite of the Itarare Group (CarboniferousPermian), Paraná Basin, Brasil. Am. Assoc. Petrol. Geol. Bull., 75(1):62-85

BOUMA, A.H. 1962. Sedimentology of Some Flysch Deposits. Amsterdam, Elsevier. $168 \mathrm{p}$.

DE SANTA ANA, H. 1989. Consideraciones tectónicas y deposicionales de la Cuenca Norte Uruguaya. Bol. Tec. ARPEL, 18(4):319-339.

EYLES, N.; EYLES, C.H.; MIALL, A. 1983. Lithofacies types and vertical profiles models; an alternativo approach to the description and environment interpretation of glacial diamict and diamictites sequences. Sedimentology, 30:393-410.

FALCONER, J.D. 1937. La Formación de Gondwana en el Nordeste del Uruguay, con Especial Referencia a los Terrenos Eogondwánicos. Montevideo, Inst. Geol. Perf. (Boletín 23).

FERRANDO, L. \& ANNORÉIS, R.R. 1986. Nueva estratigrafía en el Gondwana del Uruguay. In: CONGR. LAT. HIDROC, 1. Buenos Aires, 1986. Actas... Buenos Aires, ARPEL. v. 1, p. 295-323.

FERRANDO, L. \& MONTANA, J.R. 1987. Hipótesis preliminar sobre la evolución paleogeográfica de la Cuenca de Paraná en el Uruguay. In: SIMP. SUL-BRAS. GEOL., 3. Curitiba, 1987. Ato... Curitiba, SBG. v. 1, p. 393-400.

GAMA, E., Jr.; PERINOTTO, f; RIBEIRO, H.; PADULA, E. 1989. Análise de Facies em Depósitos de Retrabalhamento Glacial do SubGrupo Itararé: um Guia de Campo. Rio Claro, IGCE-UNESP. 88 p.

LOWE, D.R. 1979. Sediment gravity flows: their classification and some problems of application to natural flows. In: DOYLLE, L.J. \& PILKEY, O.H. eds. Geol Cont. Slopes, SEPM Spec. Publ, 27:75-82.

MARQUES-TOIGO, M. 1988. Palinologia, Bioestratigrafia e Paleoecologia do Neopaleozóico da Bada do Paraná nos Estados do Rio Grande do Sul e Santa Catarina, Brasil. Porto Alegre. 259 p. (Tese de Doutoramento, IG/UFRGS).
MIALL, A.D. 1983. Glaciomarine sedimentation in the Gowganda Formation (Uronian), Nothern Ontario. J. Sediment Petrol, 53:477-491.

MIALL, A.D. 1985. Principies of Sedimentary Basin Analysis. $2^{\mathrm{a}}$ ed. New York, Springer-Verlag. $490 \mathrm{p}$.

MIDDLETON, G.B. \& HAMPTON, M.A. 1973. Sediment gravity flows: mechantes of flows and deposition. In: Turbidites and Deepwater sedimentation. SEPM Pacif. Section Short Course Lect., Notes; $38 \mathrm{p}$.

REMY, W. 1975. The glacial changes at the Carboniferous-Permian Boundary in Europe and North America. In: LE. WHITE MEMORIAL SYMP. "The Age of the Dunkrard", 1. Norgantown, 1972. Proceedings... Norgantown. p. 305-343.

RUSSO, A; ARCHANGELSKY, S.; GAMERRO, J.C. 1980. Los depósitos suprapaleozoicos en el subsuelo de la llanura Chaco-Paraná, Argentina. In: CONGR. ARG. PALEONT. Y BIOESTR. 2 y CONGR. LATINOAM. PALEONT., 1. Buenos Aires, 1980. Actas... Buenos Aires, APA. v. 4, p. 157-173.

STEWART, W.N. 1983. Paleobotany and the Evolution of Plants. New York, Cambridge University Press. $405 \mathrm{p}$.

TERRA AROCENA, E. 1926. Nota sobre el Piso Itararé y los Esquistos Marinos de Rincon de Alonso. Montevideo, Inst. Geol. Perf. p. 8-20. (Boletín 8).

WALKER, R.G. 1984. Facies Models. 2 ed. Toronto, Geol. Assoc. Can. $211 \mathrm{p}$.

WALTHER, K. 1924. Los Resultados de las Perforaciones en el Departamento de Cerro Largo, en Bancos de Carbón de Piedra. Montevideo, Inst. Geol. Perf. (Boletín 6)

WHITE, I.C. 1908. Relatórío sobre as Coal Measures e Rochas Associadas ao sul do Brasil. Rio de Janeiro, Comissão dos Estudos das Minas de Carvão. 300 p. (Relatório Final, Parte 1).

MANUSCRITO A759

Recebidoem 5 denudo de 1993 Revisáo do autor em 9 de agosto de 1994 Revisáo aceita em 31 de agosto de 1994 